\title{
Comparison of the Fractionation Efficiencies of Elution Method and Precipitation Chromatography of Polymer Fractionation
}

\author{
Minoru UEDA \\ Central Research Laboratories, Kuraray Co., Ltd., \\ Sakazu, Kurashiki, Japan.
}

(Received July 1, 1971)

\begin{abstract}
Comparison of fractionation efficiencies of elution method and precipitation chromatography was made on a conventional polystyrene. Molecular weight distributions obtained from fractionation data were in close agreement with each other. The maximum molecular weight of these fractionations, however, was higher in the precipitation chromatography than in the elution method which indicates better resolution in the former than in the latter. But this difference was inverted when the elution method was applied to the identical sample but treated with acetylating agent. The molecular weight distributions of the representative fractions obtained by the two methods as measured by sedimentation velocity method at a theta condition were essentially symmetrical with respect to their centroid and had $M_{w} / M_{n}$ values 1.04 to 1.06 . These results obtained for fractions prepared by elution fractionation were qualitatively in agreement with the theoretical results, but those obtained by precipitation chromatography were far broader than that expected from the elementary consideration of the principle. Possible reasons of this discrepancy are considered.

KEY WORDS Polystyrene / Elution Method / Precipitation Chromatography / Fractionation Efficiency / Sedimentation / $\theta$ Condition / Molecular Weight Distribution / Acetylation /
\end{abstract}

As regards the elementary consideration of the principles of fractionation, the efficiency of the precipitation chromatography developed by Baker and Williams ${ }^{1}$ must be superior to the elution method and all methods based on the solubility behavior of polymers. This is because the precipitation chromatography is considered to do a multi-stage fractionation. Under actual conditions, however, various factors may cause lowering of the resolution of the precipitation chromatography. ${ }^{2}$ Unfortunately, due to experimental difficulties, there is as yet no direct evidence whether precipitation chromatography actually permits a multi-stage fractionation or how much the actual fractionation deviates from the ideal fractionation.

Comparison of the elution method and the precipitation chromatography has been made by a few authors principally by comparing molecular weight distributions of samples obtained by these two methods. Hall, ${ }^{3}$ and Schneider, et al., ${ }^{2}$ compared the mass distribution curves of conventional and high molecular weight poly- styrene samples and found that the difference between the two methods was not so great as one might expect from elementary considerations. Guillet, et al., ${ }^{4}$ compared both mass distribution curves and sedimentation velocity patterns for fractions of polyethylene and found that the precipitation chromatography was more effective than the elution method. However, polyethylene is rather unique in the field of column fractionation. ${ }^{5}$ Cantow, et al. ${ }^{6}$ examined by analytical fractionation of the fractions obtained by largescale precipitation chromatography and found that the fractions were extremely narrow. However, the Schulz-Dinglinger procedure ${ }^{7}$ may no longer be applied to such narrow fractions. ${ }^{8-9}$

In the present study, we have first compared mass distribution curves of a conventional polystyrene sample obtained by the two methods by using the apparatus and procedures appropriate for each method and then analyzed representative fractions by sedimentation transport measurements under a theta condition. 


\section{EXPERIMENTAL}

\section{Material}

A polystyrene S-D1, which was supplied from the Committee of Molecular Weight and Molecular Weight and Molecular Weight Distribution of the Society of Polymer Science, Japan was selected as the sample. Average value of intrinsic viscosity $[\eta]$ in toluence at $30^{\circ} \mathrm{C}$ was $1.15 \mathrm{dl} / \mathrm{g} .{ }^{10}$

The reagent grade solvents were purified to the extent necessary to carry out each experiment.

\section{Column Elution Fractionation}

The column used in the elution fractionation was approximately $50 \mathrm{~cm}$ long and $4.0 \mathrm{~cm}$ in inner diameter, and fitted with a medium porosity sintered glass disc near the bottom, a glass joint at the top and a glass cock at the bottom to control the flow rate of the eluant. The column was also fitted with a glass jacket so that thermostated water can be circulated along its length to maintain a desired temperature. Thermostated solvents were supplied onto the column through a Dimroth-type condenser, the outer part of which was circulated with thermostated water at the same temperature as the column. Glass beads of $0.1-\mathrm{mm}$ diameter cleaned by a procedure analogous to that proposed by Jungnickel, et al., ${ }^{11}$ were used as the support of polymer.

About two grams of polystyrene S-D1 was dissolved in 180-m $l$ benzene, and after adding $800 \mathrm{~g}$ of glass beads the solvent was evaporated at a slightly elevated temperature. After drying, the beads were passed through a No. 50 seive, and placed in the column by the wet method. After standing overnight, elution was made with either methyl ethyl ketone or benzene as solvent and ethanol as precipitant at a flow rate of $250 \mathrm{ml}$ / $\mathrm{hr}$ at $30^{\circ} \mathrm{C}$. Fractionation was performed by batchwise variation of the solvent-precipitant mixture. Volume of each solvent mixture was $250 \mathrm{~m} l$ which was 1.4 times as large as the holdup of the column. This amount of solvent was sufficient for the present purpose, because mutual diffusion of the two adjacent solvent mixtures may largely be restricted by the existance of glass beads.

\section{Precipitation Chromatography}

The precipitation chromatography was carried out by the apparatus and the procedure employed previously. ${ }^{8}$

All fractions obtained were recovered by evaporating volatiles, redissolved in benzene, filtered through a No. 3 sintered glass disc into a tared test tube, followed by evaporation of benzene, and finally dried to constant weight in vacuo at $80^{\circ} \mathrm{C}$. Some fractions were again dissolved and reprecipitated.

\section{Viscosity}

Dillute solution viscosities were measured either at four concentrations or at a single concentration (usually 0.3 to $0.5 \mathrm{~g} / \mathrm{d} l$ ) in toluene at $30^{\circ} \mathrm{C}$. In the case of single point determination, $[\eta]$ was calculated from the relative viscosity $\eta_{r}$ by using Wenger's equation. ${ }^{12}$

Molecular weight $M$ of each fraction was es-

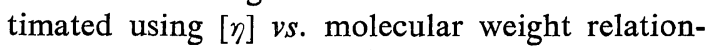
ship given by Kawahara ${ }^{13}$

$$
[\eta]=7.9 \times 10^{-4} M^{1 / 2}+15.8 \times 10^{-7} M
$$

\section{Sedimentation Transport}

Sedimentation measurements were carried out by the method reported previously ${ }^{8}$ either at four concentrations or at a single concentration. Data obtained at a single concentration were extrapolated to infinite dilution in terms of the relation of Pyun and Fixman. ${ }^{14}$ Distribution of the sedimentation coefficient of fractions corrected for diffusion and concentration effects were transformed into molecular weight distributions by using the following equation ${ }^{8}$

$$
S=1.32 \times 10^{-15} M^{1 / 2}
$$

where $S$ is the sedimentation constant in second.

\section{RESULTS AND DISCUSSION}

\section{Fractionation of Whole Polymer}

Table I and II summarize the fractionation data on polystyrene S-D1 obtained from the elution method and precipitation chromatography, respectively. The recovery of the polymer was essentially complete in both methods. The sum

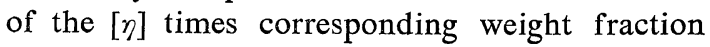
is about $1 \%$ smaller than the value of the original sample. Integral molecular weight distributions obtained from the data listed in Tables I and II using the usual Schulz-Dinglinger pro- 
Table I. Fractionation data from precipitation chromatography experiment on sample S-D1 ${ }^{\text {a }}$

\begin{tabular}{|c|c|c|c|c|}
\hline $\begin{array}{l}\text { Fraction } \\
\text { no. }\end{array}$ & $\begin{array}{l}\text { Weight, } \\
\text { mg }\end{array}$ & $\begin{array}{c}\text { Weight, } \\
\%\end{array}$ & $\begin{array}{l}{[\eta],} \\
\mathrm{d} l / \mathrm{g}\end{array}$ & $M \times 10^{-4}$ \\
\hline $16-20$ & 37.1 & 1.49 & 0.192 & 3.2 \\
\hline $21-22$ & 36.3 & 1.46 & 0.246 & 4.7 \\
\hline 23 & 23.6 & 0.95 & 0.263 & 5.2 \\
\hline 24 & 31.5 & 1.27 & 0.325 & 7.2 \\
\hline 25 & 24.5 & 0.99 & 0.337 & 7.6 \\
\hline 26 & 25.1 & 1.01 & 0.347 & 7.9 \\
\hline 27 & 28.6 & 1.15 & 0.375 & 8.9 \\
\hline 28 & 36.1 & 1.46 & 0.412 & 10.2 \\
\hline 29 & 40.8 & 1.64 & 0.439 & 11.1 \\
\hline 30 & 33.1 & 1.33 & 0.474 & 12.4 \\
\hline 31 & 49.7 & 2.00 & 0.496 & 13.2 \\
\hline 32 & 56.0 & 2.26 & 0.480 & 12.6 \\
\hline 33 & 65.4 & 2.64 & 0.519 & 13.2 \\
\hline 34 & 70.9 & 2.86 & 0.558 & 15.6 \\
\hline 35 & 59.8 & 2.41 & 0.618 & 17.9 \\
\hline 36 & 60.1 & 2.42 & 0.656 & 19.5 \\
\hline 37 & 64.8 & 2.61 & 0.673 & 20.1 \\
\hline 38 & 26.9 & 1.08 & 0.724 & 22.2 \\
\hline 39 & 75.5 & 3.04 & 0.739 & 22.9 \\
\hline 40 & 85.9 & 3.46 & 0.743 & 23.0 \\
\hline 41 & 90.9 & 3.66 & 0.790 & 25.0 \\
\hline 42 & 92.4 & 3.72 & 0.841 & 27.2 \\
\hline 43 & 85.5 & 3.45 & 0.857 & 27.9 \\
\hline 44 & 95.0 & 3.83 & 0.942 & 31.5 \\
\hline 45 & 93.4 & 3.76 & 1.00 & 34.1 \\
\hline 46 & 89.7 & 3.61 & 1.08 & 37.7 \\
\hline 47 & 89.6 & 3.61 & 1.13 & 39.9 \\
\hline 48 & 87.8 & 3.54 & 1.20 & 43.1 \\
\hline 49 & 86.4 & 3.48 & 1.29 & 47.3 \\
\hline 50 & 89.0 & 3.56 & 1.39 & 51.9 \\
\hline 51 & 98.0 & 3.95 & 1.47 & 55.7 \\
\hline 52 & 107.9 & 4.35 & 1.57 & 60.5 \\
\hline 53 & 99.6 & 4.01 & 1.68 & 65.8 \\
\hline 54 & 83.7 & 3.37 & 1.89 & 76.0 \\
\hline 55 & 89.2 & 3.59 & 2.09 & 85.9 \\
\hline 56 & 76.7 & 3.09 & 2.11 & 86.9 \\
\hline 57 & 45.5 & 1.83 & 2.76 & 120 \\
\hline 58 & 32.8 & 1.32 & 3.18 & 142 \\
\hline 59 & 14.9 & - & - & - \\
\hline $60-61$ & 2.2 & - & - & - \\
\hline
\end{tabular}

a Polymer loading, $2.470 \mathrm{~g}$ of sample onto $250 \mathrm{~g}$ of glass beads from benzene solution by slow evapolation of solvent with stirring to prevent flocculation of beads by polymer; column, $5 \times$ $60 \mathrm{~cm}$, packed with $0.1-\mathrm{mm}$ glass beads; temperature gradient, 60 to $10^{\circ} \mathrm{C}$; volume of solvent reservior, 1.11; solvent gradient, 100-vol $\%$ MEK to absolute ethanol; flow rate, below $25 \mathrm{ml} / \mathrm{hr}$ from fraction 12; volume of fraction, 30 to $35 \mathrm{ml}$; total weight of fractions, $2.482 \mathrm{~g}$. cedure $^{7}$ and the differential mass distribution obtained by graphical differentiation of the corresponding integral distribution are shown in Figures 1 and 2. These curves are in close agreement with each other and similar to those obtained carefully in many laboratories of Japan for the identical sample by various experimental methods. ${ }^{10}$

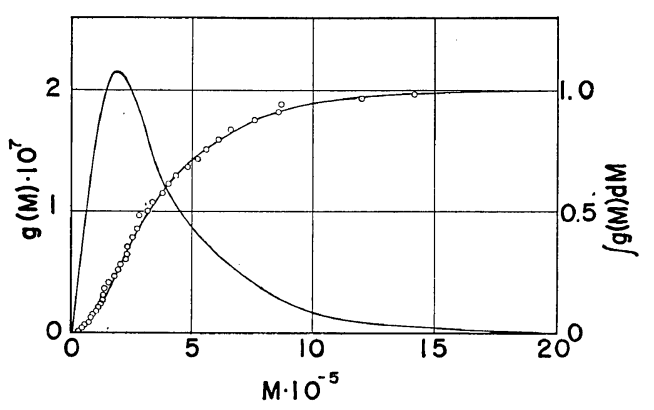

Figure 1. Integral mass distribution for polystyrene S-D1 obtained by appling the usual SchulzDinglinger procedure to the data of precipitation chromatography in Table I and differential mass distribution obtained by graphical differentiation of the integral distribution.

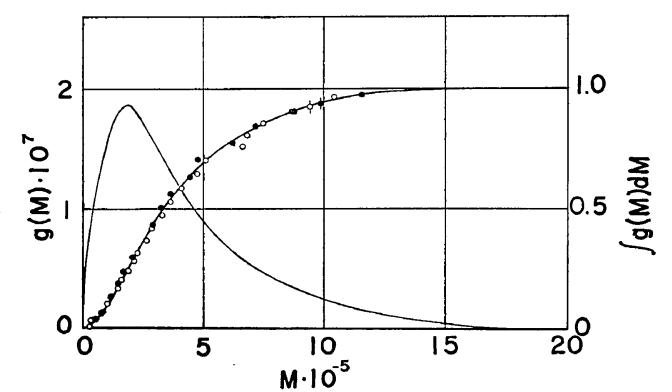

Figure 2. Integral mass distribution for S-D1 obtained by applying Schulz-Dinglinger procedure to the data of two experiments of column elution fractionation (Table II) and differential mass distribution obtained therefrom: $\bigcirc$, results from Table IIa; $\bigcirc$, results from Table IIb. Molecular weights are arranged in increasing order. The fractions inverted in $[\eta]$ are indicated by pip.

In the precipitation chromatography experiment the square root of the highest molecular weight fraction times the concentration in $\mathrm{g} / \mathrm{d} l$ is about 370. This satisfies the condition proposed by Schneider, et al. ${ }^{2}$ They predicted for a conven- 


\section{UEDA}

Table IIa. ${ }^{a}$ Fractionation data from elution fractionation on sample S-D1

\begin{tabular}{|c|c|c|c|c|c|}
\hline $\begin{array}{c}\text { Fraction } \\
\text { no. }\end{array}$ & $\begin{array}{c}\text { Solvent mixture } \\
\text { MEK/(MEK + EtOH }) \\
\text { by volume }\end{array}$ & $\begin{array}{l}\text { Weight } \\
\text { mg }\end{array}$ & Weight, $\%$ & $\begin{array}{c}{[\eta],} \\
\mathrm{ml} / \mathrm{g}\end{array}$ & $M \times 10^{-4}$ \\
\hline $\begin{array}{l}1 \\
2 \\
3 \\
4^{b} \\
5\end{array}$ & $\begin{array}{l}58.0 \\
66.0 \\
69.0 \\
71.0 \\
73.0\end{array}$ & $\begin{array}{r}5.0 \\
38.0 \\
61.8 \\
63.4 \\
80.5\end{array}$ & $\begin{array}{l}0.155 \\
1.75 \\
3.41 \\
2.92 \\
3.71\end{array}$ & $\begin{array}{l}-\overline{183} \\
0.249 \\
0.338 \\
0.395\end{array}$ & $\begin{array}{l}- \\
3.0 \\
4.9 \\
7.6 \\
9.7\end{array}$ \\
\hline $\begin{array}{c}6 \\
7 \\
8^{b} \\
9 \\
10\end{array}$ & $\begin{array}{l}74.0 \\
75.0 \\
76.0 \\
77.0 \\
77.5\end{array}$ & $\begin{array}{l}104.7 \\
113.0 \\
102.0 \\
146.3 \\
152.4\end{array}$ & $\begin{array}{l}4.83 \\
5.21 \\
4.70 \\
6.74 \\
7.01\end{array}$ & $\begin{array}{l}0.450 \\
0.531 \\
0.578 \\
0.677 \\
0.721\end{array}$ & $\begin{array}{l}11.6 \\
14.3 \\
16.5 \\
20.5 \\
22.3\end{array}$ \\
\hline $\begin{array}{l}11 \\
12^{b} \\
13 \\
14 \\
15^{b}\end{array}$ & $\begin{array}{l}78.0 \\
78.5 \\
79.0 \\
79.5 \\
80.0\end{array}$ & $\begin{array}{l}156.9 \\
133.0 \\
140.1 \\
147.1 \\
167.6\end{array}$ & $\begin{array}{l}7.23 \\
6.13 \\
6.46 \\
6.78 \\
7.72\end{array}$ & $\begin{array}{l}0.884 \\
0.963 \\
1.065 \\
1.222 \\
1.287\end{array}$ & $\begin{array}{l}29.0 \\
32.5 \\
36.3 \\
44.2 \\
47.3\end{array}$ \\
\hline $\begin{array}{l}16 \\
17^{\mathrm{b}} \\
18 \\
19 \\
20\end{array}$ & $\begin{array}{l}80.5 \\
81.0 \\
81.5 \\
82.0 \\
82.5\end{array}$ & $\begin{array}{r}143.5 \\
150.6 \\
117.5 \\
80.2 \\
43.6\end{array}$ & $\begin{array}{l}6.61 \\
6.94 \\
5.42 \\
3.70 \\
2.01\end{array}$ & $\begin{array}{l}1.596 \\
1.802 \\
2.118 \\
2.670 \\
2.360\end{array}$ & $\begin{array}{c}62.0 \\
71.8 \\
87.5 \\
116 \\
98.6\end{array}$ \\
\hline 21 & 100 & 22.9 & 1.06 & - & - \\
\hline
\end{tabular}

a Initial sample loading, $2.211 \mathrm{~g}$; solvent, MEK; precipitant, ethanol; temp, $30^{\circ} \mathrm{C}$; sum of weights of fractions, $2.170 \mathrm{~g}$.

b Used for solubility test shown in Figure 6.

Table IIb. ${ }^{a}$ Fractionation data from elution fractionation on sample S-D1

\begin{tabular}{cccccr}
\hline $\begin{array}{c}\text { Fraction } \\
\text { no. }\end{array}$ & $\begin{array}{c}\text { Solvent mixture } \\
\text { Benzene/(Benzene+EtOH), } \\
\text { by volume }\end{array}$ & $\begin{array}{c}\text { Weight, } \\
\text { mg }\end{array}$ & $\begin{array}{c}\text { Weight, } \\
\%\end{array}$ & $\begin{array}{c}{[\eta]} \\
\mathrm{d} l / \mathrm{g}\end{array}$ & $M \times 10^{-4}$ \\
\hline 1 & 0.280 & 34.9 & 1.88 & 0.176 & 2.9 \\
2 & 0.300 & 52.6 & 2.84 & 0.209 & 3.6 \\
3 & 0.320 & 64.6 & 3.49 & 0.343 & 8.0 \\
4 & 0.330 & 69.3 & 3.74 & 0.410 & 10.2 \\
5 & 0.335 & 60.7 & 3.28 & 0.461 & 12.0 \\
6 & 0.340 & 60.7 & 3.28 & 0.540 & 15.0 \\
7 & 0.345 & 67.7 & 3.65 & 0.561 & 15.7 \\
8 & 0.350 & 74.4 & 4.01 & 0.645 & 19.2 \\
9 & 0.354 & 82.6 & 4.46 & 0.702 & 21.5 \\
10 & 0.357 & 80.6 & 4.35 & 0.770 & 24.2 \\
11 & 0.360 & 86.0 & 4.64 & 0.821 & 26.5 \\
12 & 0.364 & 96.7 & 5.22 & 0.878 & 28.9 \\
13 & 0.367 & 105.6 & 5.70 & 0.977 & 33.0 \\
14 & 0.370 & 102.2 & 5.51 & 1.05 & 36.3 \\
15 & 0.3725 & 110.0 & 5.93 & 1.15 & 40.7 \\
16 & 0.375 & 110.9 & 5.95 & 1.29 & 47.3 \\
17 & $0.377_{5}$ & 99.6 & 5.37 & 1.37 & 51.0 \\
18 & 0.380 & 91.3 & 4.93 & 1.73 & 68.3 \\
19 & 0.382 & 97.9 & 5.28 & 1.69 & 66.3 \\
20 & 0.384 & 89.7 & 4.84 & 1.86 & 74.7 \\
21 & 0.386 & 77.1 & 4.16 & 2.11 & 87.0 \\
22 & 0.388 & 56.5 & 3.05 & 2.49 & 104 \\
23 & 0.390 & 33.6 & 2.62 & 2.50 & 105 \\
24 & 0.392 & 1.81 & 2.24 & 93.8 \\
\hline
\end{tabular}

a Initial sample loading, $1.871 \mathrm{~g}$; solvent, benzene; precipitant, ethanol; temp, $30^{\circ} \mathrm{C}$; sum of weights of fractions, $1.854 \mathrm{~g}$. 
tional polystyrene of moderate molecular weight that this product should be smaller than about 400 for a successful fractionation. The maximum [ $\eta]$, which was used by several authors as the measure of relative efficiency of fractionation, is 3.18 for precipitation chromatography. This is almost the same as the highest molecular weight $([\eta]=3.37 \mathrm{~d} l / \mathrm{g})$ obtained by Kawahara on the identical sample using repeated fractional precipitation of the first of 35 fractions which had been obtained by successive precipitation with the system toluene-poly(ethylene glycol) at the initial concentration of $0.27 \mathrm{~g} / \mathrm{d} l .^{15}$ Thus we may expect that the highest molecular weight obtained by the present precipitation chromatography is nearly the highest molecular weight obtainable experimentally. This contrasts with the results of $\mathrm{Hall}^{3}{ }^{3}$ who obtained $5.34 \mathrm{~d} l / g$ for the $[\eta]$ of the first fraction fractionated by successive precipitation, while the value for the final fraction isolated by the precipitation chromatography was $4.15 \mathrm{~d} l / g$. It seems likely that the difference results from the difference in fractionation conditions of the precipitation chromatography and not from the difference in average molecular weight of the polymer sample fractionated. Molecular weight inversion, which is often observed in the column elution fractionation, was not observed in the present results of precipitation chromatography.

In the column elution experiment, $[\eta]$ of the highest molecular weight fraction was 2.67 and $2.50 \mathrm{~d} l / g$ for the solvent system MEK-ethanol and benzene-ethanol, respectively. Inversion of intrinsic viscosity in the highest molecular weight region occured in both cases to nearly equal extent. On the basis of the proposal ${ }^{3}$ that the highest intrinsic viscosity can be used as a measure of the efficiency of a fractionation, the elution method achieves poorer resolution than the precipitation chromatography. This is essentially consistent with the results obtained by Schneider, et al., ${ }^{2}$ although rather different experimental conditions were adopted for the elution fractionation. They attributed the origin of the difference in performance of the two methods to the adsorption of trace amount of hydroxy groups present in a part of species of the polymer onto glass beads. They made this conclusion from the fact that no molecular weight inversion was observed when fractionation was carried out on acetylated sample of the same polystyrene and that adsorption of slightly hydrolyzed poly(vinyl acetate) on iron powder is far greater in amount and force than the pure poly (vinyl acetate). ${ }^{16}$ Similarity of behavior in the final fractions in the present study with those of Schneider, et al., might suggest that the origin of the apparent inferiority of the elution method to the precipitation chromatography could be attributed to the same reason. To examine this possibility, we also performed an additional elution experiment on acetylated polystrene S-D1. Acetylation was carried out under the condition used to acetylate polystyrene prepared by emulsion polymerization. ${ }^{17}$ Integral molecular weight distribution obtained from the fractionation data is shown in Figure 3 by open circles, which

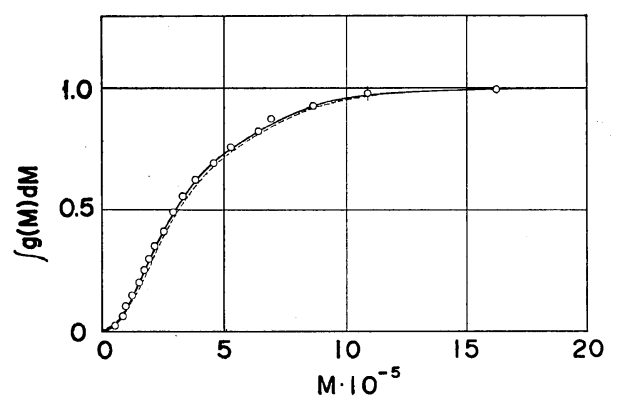

Figure 3. Integral mass distribution for acetylated polystyrene S-D1 obtained by the usual method to data obtained by elution fractionation. Fractionation conditions were essentially the same as those described in Table IIa.

can favorably be compared with those obtained by precipitation chromatography data shown by chain line. The highest intrinsic viscosity increased to $3.57 \mathrm{~d} l / g$ indicating extensive improvement of the elution method. This is the highest value in this sample among the values obtained by several laboratories of Japan. ${ }^{10}$ Inversion of intrinsic viscosity, however, did not vanish though diminished extensively.

These facts seem to indicate that the molecular weight inversion as expressed by intrinsic viscosity, which was observed frequently in the final stage of the column elution method, re- 
sulted mainly from the adsorption of polymer species containing trace of polar or hydrogen bonding groups as impurities onto the glass beads. It is well known that glass surface is surface active to some extent. ${ }^{18}$ Actually, almost no elution of polymer occured when we tried to fractionate poly (vinyl alcohol) by the elution method above $65^{\circ} \mathrm{C}$ using water as solvent and $n$-propanol as precipitant. ${ }^{19}$ The origin of the residual slight inversion in intrinsic viscosity is not clear to us. If acetylation was complete, the reason other than interaction between polymer and glass beads must be sought. The only possible origin for this phenomenon seems to be the formation of aggregates of glass beads. Observation under a low-power microscope suggested that a part of polymer deposited occured in the interspace between the beads. Complete elimination of this phenomenon was not possible in the present study. In this connection lack of this abnormal effect in precipitation chromatography is surprising, since much extensive aggregation of glass beads by polymer due to much greater polymer deposition onto the unit volume of glass beads. A possible explanation of this apparently unreasonable problem is that both polymer adsoaption onto glass surface and/or delay of diffusion of polymer species from glass surface to dilute phase vanish almost completely at elevated temperatures, i.g., $60^{\circ} \mathrm{C}$.

Therefore, incorporation of higher operating temperature with treatment of glass beads, for example, by dimethyl silicon dichloride to deactivate adsorption capability of glass surface ${ }^{20}$ may contribute largely to eliminate the molecular weight inversion in the elution fractionation of polymers. Unfortunately, this possibility has not been examined yet. In any event, the above difference in the final stage of fractionation found between elution method and precipitation chromatography may not necessarily be attributed to the difference of the principle of fractionation but rather to the trace of chemical heterogeneity or anormalous reaction of the polymer. Thus, in order to compare the relative efficiency, molecular weight distribution of fractions obtained by the two methods should be compared.

\section{Comparison by Sedimentation Velocity}

Figure 4 represents the final results of sedimentation analysis for three fractions obtained by a precipitation chromatography experiment (designated as $\mathrm{P}$ ) and four from an elution experiment (designated as E). Weight- and number-average

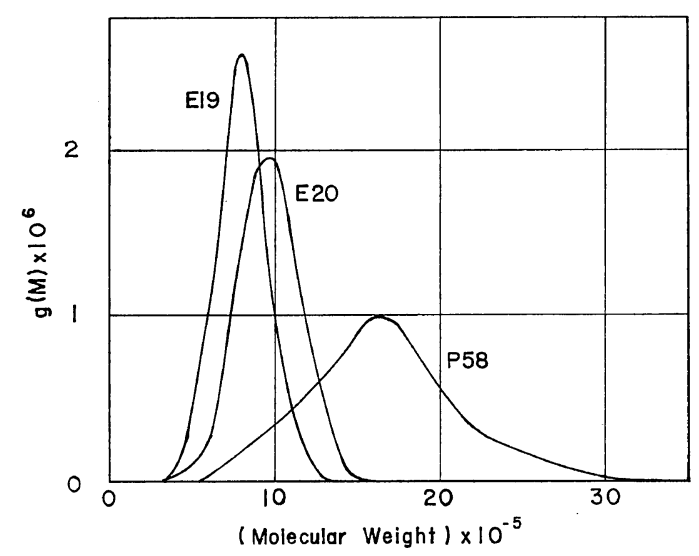

(a)

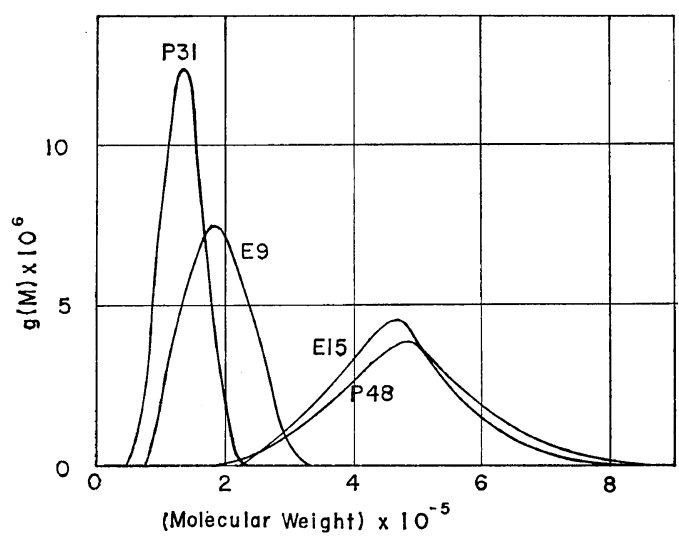

(b)

Figure 4. Differential molecular weight distributions of fractions obtained by precipitation chromatography (designated as $\mathrm{P}$ ) and those by elution method (designated as E).

molecular weights calculated from these molecular weight distribution curves are summarized in Table III together with their intrinsic viscosities in toluene at $30^{\circ} \mathrm{C}$, viscosity-average molecular weights $M_{v}$, and apparent sedimentation coefficients $\bar{S}$ defined in terms of the maximum refractive index gradient. Except for the fractions E-19 and P-58, $M_{v}$ values are in close agreement with the corresponding $M_{w}$ 's. Since [ $\left.\eta\right]$ of E-19 
Comparison of the Fractionation Efficiencies

Table III. Results of sedimentation analysis

\begin{tabular}{crccccc}
\hline $\begin{array}{c}\text { Sample } \\
\text { no }\end{array}$ & $\bar{S}^{\mathrm{a}}$ & $M_{n} \times 10^{-4}$ & $M_{w} \times 10^{-4}$ & $M_{w} / M_{n}$ & {$[\eta]$} & $M_{v} \times 10^{-4}$ \\
\hline E-9 & 5.7 & 17.8 & 18.9 & 1.06 & 0.68 & 20.5 \\
E-15 & 8.9 & 43.8 & 46.5 & 1.06 & 1.29 & 47.3 \\
E-19 & 11.8 & 77.2 & 80.5 & 1.04 & 2.67 & 116 \\
E-20 & 13.0 & 91.5 & 95.9 & 1.04 & 2.36 & 98.6 \\
P-31 & 4.9 & 13.0 & 13.6 & 1.05 & 0.50 & 13.2 \\
P-48 & 9.2 & 45.9 & 47.9 & 1.04 & 1.20 & 43.1 \\
P-58 & 16.8 & 150.9 & 164.2 & 1.09 & 3.18 & 142 \\
\hline
\end{tabular}

a $\bar{S}$ stands for Svedberg unit.

and E-20 was reproducible, the inconsistency between $M_{w}$ and $M_{v}$ of these fractions seems to suggest the existence of some anomalous effect at least in one of these fractions. The reason of this phenomenon remains unlcear. Doublelogarithmic plots of $[\eta]$ and $\bar{S}$ listed in Table III together with those obtained by McCormick, ${ }^{12}$ which are not represented here, have shown that the data point of E-58 deviates from the linear relationship obtained by the least-squares method. The extent of the deviation corresponded to the difference between $M_{v}$ and $M_{w}$ of P-58. Although eq 2 has been established for $8.5 \times 10^{4}<M_{w}<$ $41.5 \times 10^{4}$, Berry's data ${ }^{22}$ suggests that the equation can be extended the upper limit to $1.8 \times 10^{6}$. Thus the difference in $M_{w}$ and $M_{v}$ of P-58 may be attributed to the experimental error of at least one of these values. The ratio $M_{w} / M_{n}$ calculated from these curves are 1.04 to 1.06 (except for the last fraction from precipitation chromatography, the value for this fraction is 1.09) irrespective of the fractionation method. All distributions are nearly symmetrical with respect to their centroid. These facts indicate that the fractionation efficiency of precipitation chromatography and elution method are essentially the same if these fractionations were carried out by applying conditions used in the present study and usually used for these methods. These are rather unexpected results at least from the simple elementary considerations of these methods. But prior to presenting considerations about the source of these phenomena, the fractionation efficiency of the elution method will be discussed.

From the principle of fractionation, the elution method can be regarded as a form of fractional solution. If the elution method truly carries out fractional solution, the results obtained by this method should be essentially consistent with those obtained by model fractionation because such comparison was shown to be valid for the examination about the efficiency of fractional precipitation..$^{23-25}$ Such calculations were made by several authors. ${ }^{26-29}$ But the fractionation conditions in the present study resembles with those of Matsumoto, et al. ${ }^{26}$ In addition, in spite of the neglect of the removal of dissolved polymer species from concentrated phase, the $M_{w} / M_{n}$ values obtained by them are qualitatively in close agreement with those obtained by Koningsveld. ${ }^{28}$ Thus we may compare the present results of the elution experiment with those obtained by Matsumoto, et al. Their calculation consisted of applying Flory's solubility relationship ${ }^{30}$

$$
f_{x}=\frac{1}{1+R \exp (A x)}
$$

to a polymer with $x_{n}=500$ having Schulz' distribution $^{31}$

$$
g(x)=\frac{x}{x_{n}{ }^{2}} \exp \left(-x / x_{n}\right)
$$

Here, $f_{x}$ is the fraction of the polymer molecule of chain length $x$ remaining in the more dilute phase, $R$ is the ratio of the volumes of the precipitate or swollen gel and supernatent phases, and $A$ is a complex function of Flory's solubility parameter. Thus calculated series of eight fractions, assuming $10^{-2}$ for $R$, were essentially symmetrical with respect to their peaks and had $x_{w} / x_{n}$ values nearly equal or less than 1.1 except the first fraction. These features of a model 
calculation are just seen in the representative fractions obtained by the elution method. In addition, the solutions collected were obtained as essentially saturated. This was demonstrated by the appearance of polymer precipitate from eluted solution by slight temperature depression from that used in the fractionation procedure or addition of a few drops of precipitant to total volume of solution at the same as the operating temperature. Also, as shown in Figure 5, sol-

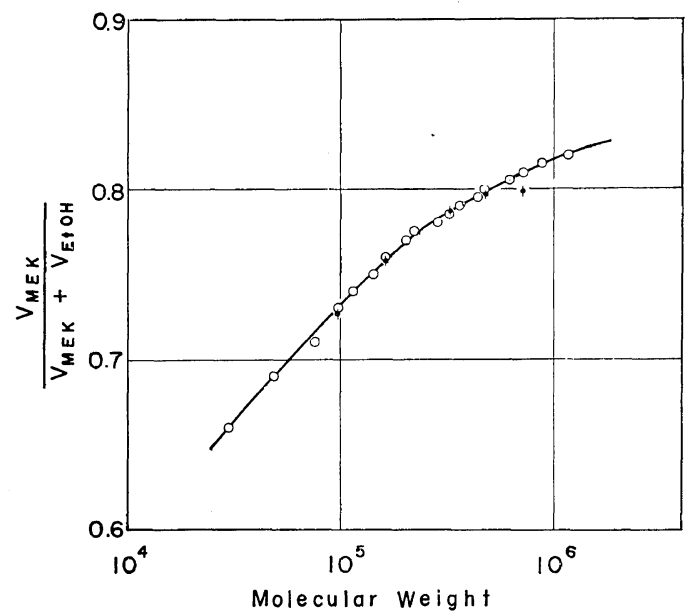

Figure 5. Comparison of polystyrene molecular weight $v$ s. solvent composition: $\bigcirc$, data from the elution method; data from titration of MEK solution of the corresponding fractions with ethanol.

vent composition vs. molecular weight relationship obtained from the elution experiment was in close agreement with that obtained by precipitation experiments made at the same polymer concentrations at the initial precipitation points as those of the eluted solution. It may be concluded form these considerations that the elution method is truly a form of fractional solution. Similar results were obtained for fractions of poly (vinyl acetate) obtained by large-scale fractionation using techniques similar to those applied for IUPAC living polystyrene by Homma, et al. ${ }^{8}$ But an appreciably broader distribution was reported for a fraction obtained by the elution method ${ }^{5}$ when the analytical procedure similar to the present study was applied. Comparison of the two results is made in Figure 6. However, the reason of this difference is not clear

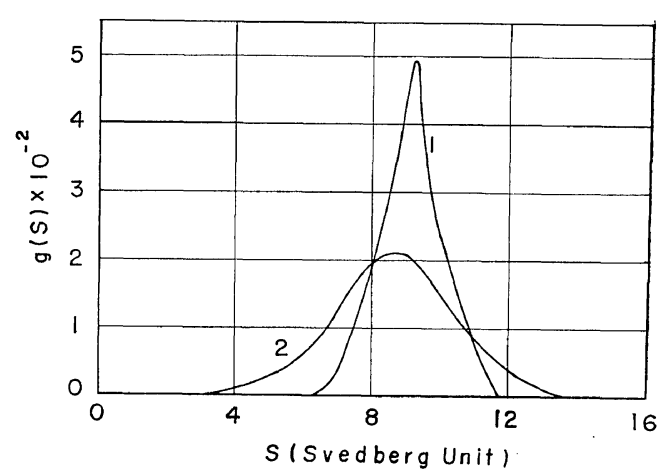

Figure 6. Comparison of $g(s)$, the distribution of sedimentation coefficient, obtained at the same solvent and temperature: curve 1, data obtained by the present study; curve 2, result obtained by Kenyon and Salyer. ${ }^{5}$

to the present author.

Elution fractionation of a sample which has a molecular weight distribution corresponding to those obtained by a elution fractionation of a conventional poly(vinyl acetate) gave a set of fractions having more homogeneity than the initial sample. ${ }^{25}$ The $M_{w} / M_{n}$ values obtained were essentiallly of the order of 1.01. Again this is essentially the same value as those obtained by fractionation of a sample having similar initial distributions by precipitation chromatography. ${ }^{8}$ Furthermore, application of elution method to a poly $(\alpha$-methylstyrene) prepared by Fujimoto, et al. ${ }^{32}$ which was shown by sedimentation velocity experiments to have extremely narrow distribution $\left(M_{w} / M_{n} \ll 1.01\right)$, was shown to give a set of fractions having successfully increasing molecular weight. ${ }^{33}$ Very recently, similar results were reported for the fractionation data obtained by applying precipitation chromatography to similar samples. ${ }^{34}$ Therefore, if the precipitation chromatography truly carries out multi-stage fractionation as was expected from the elementary consideration of principles, ${ }^{35}$ the molecular weight distribution of fractions obtained by this method should be narrower than those obtained by the above fractionation of narrow distribution samples. However, as has already been pointed out, the experimental results were in contradition with those expectation and gave fractions having distributions essentially the same as those obtained by elution 
method.

Possible reasons of the discrepancy found between the theoretical expectation and the experimental results of precipitation chromatography are (1) the deviation of the real experimental conditions from equilibrium, (2) greater loading of the sample on the unit weight of support than in the case of elution method, and (3) loading of polymer sample onto the support which occupies a rather long range of the column along the length as compared with the total length of the column. Part of the first problem has been pointed out by Schneider, et al. ${ }^{2}$ These were the filteration of polymer coacervate through the support instead of being limited to the temperature zone dictated by solvent composition and molecular weight, delay of polymer flocculation following a decrease in solvent temperature, and adsorption of polymer on the support. This last aspect was pointed out by Krigbaum and Kurz. ${ }^{36}$ In addition, delay in either the extraction process due to the finite film thickness on the surface of the supportes following the increment of solvent power or temperature of solvent may be considered to occur. Among these, other than the adsorption effect which was shown not to occur in precipitation chromatography of the present study, delay in the flocculation seems to be far greater than those in the other factors. In fact, polymer precipitates which were obtained by adding ethanol to 0.1 to 0.3 percent solution of narrow distribution polystyrene samples in MEK until precipitation point followed by lowering the temperature to about $10^{\circ} \mathrm{C}$ did not flocculate at once but needed a few to several hours to obtain a transparant dilute phase. This inclination increased rather rapidly as the initial polymer concentration decreased. Since the flow rate of effluent was 20 to $25 \mathrm{ml} / \mathrm{hr}$ and the fractions were obtained from every 30 to $35 \mathrm{ml}$ cut, the delay of the precipitate flocculation may be considered to contribute largely to the spreading of the polymer precipitates in the column. In contrast, almost no delay was observed in the phase separation by precipitation as compared with time needed to settle the precipitate onto the support. Although no direct observation was made, filteration of coacervate may therefore be of minor importance. Essential fulfill- ment of equilibrium conditions for the extraction process has already been shown for the elution method. This may be extended to the present case at least in lower temperature part other than the heated zone. It will be of value to point out in connection with the above discussion that the former is a kinetic phenomenon, the velocity of which decreases with the depression of polymer concentration, while the other factors are thermodynamic phase transitions with sufficient interfaces between the two phases produced by slight change of experimental coditions and that much severer control of experimental conditions are needed to fractionate polymer samples having narrower distributions successfully. The second problem may easily be expected from the well-known fact that the fractionation efficiency decreases appreciably as the ratio of concentrated to dilute phases increases. Actually, the polymer loading per unit weight of support was 3.5 times as much as that of the elution method. Finally, the ratio of the length of the heated zone in which the support deposited of polymer sample to the effective length of the column was about 0.1 . This is far greater than those used in the usual chromatography such as gas chromatography and gel permeation chromatography ${ }^{37}$ This factor is well-known in chromatography to diminish the fractionation efficiency appreciably. From the experimental point of view, elimination or large depression of these factors may be impractical. This is because operation at the equilibrium condition in all respects including flocculation of precipitate may need far longer time than those used in general operation and/or because large depression of polymer loading is needed to diminish the ratio of concentrated to dilute phases. Even if such fractionations were really carried out, the resulting fractions may too small to give reliable data necessary to obtain molecular weight distribution of the whole polymer and the fractions obtained. In contrast, labor which is necessary to carry out such an experiment may be terribly hard. Scale-up of the apparatus is also of impractical because control of experimental conditions becomes difficult as the dimension of the apparatus becomes larger. ${ }^{6}$ Therefore, one may conclude that the fractionation 


\section{UedA}

efficiency of precipitation chromatography which has been used in usual and is essentially the same as that of the elution method.

\section{On the Applicability of the Schulz-Dinglinger Pro- cedure}

It is seen above that the fractions obtained by elution method and the precipitation chromatography experiments have mass distributions geometrically similar and have $M_{w} / M_{n}$ values around 1.05. In the usual sence, such samples may be regarded to have very narrow distributions. The actual distributions, however, spread over a fairly wide range of molecular weight. Thus it is necessary to examine the applicability of the usual Schulz-Dinglinger procedure to the fractionation data summarized in Table I and II. Due to the essential symmetry of the mass distribution curve and the identity of $M_{w} / M_{n}$ value of representative fractions of low, middle, and highmolecular weights, one may extend these results to a series of fractions obtained by the fractionation of the present study. On the basis of one more assumption i.e., that the distributions of all fractions can be approximated by isoceles triangles corresponding to $M_{w} / M_{n}=1.05,{ }^{9}$ one may construct the differential mass distribution of the initial sample according to the procedure reported previously, ${ }^{8,9}$ i.e., sum up contributions from each fractions according to their weight fractions assuming $M_{w} / M_{n}=1.05$, which corresponds the isoceles triangular distribution of molecular weight span nearly equal to that of the peak

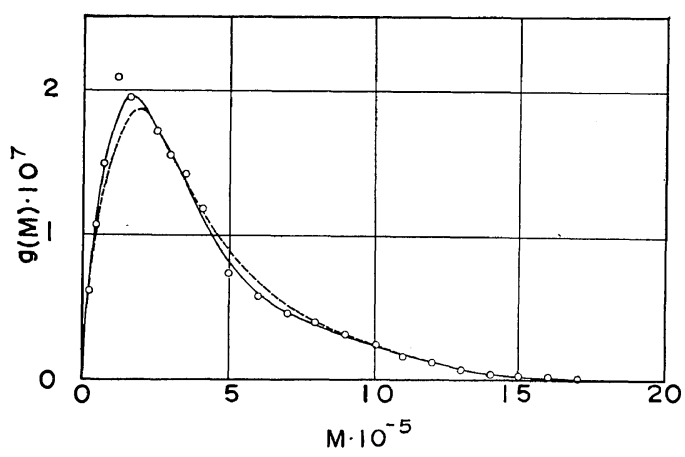

Figure 7. Comparison of mass distributions of sample S-D1 determined by applying SchulzDinglinger procedure followed by graphical differentation (chain line) and by the method described in the text (solid line and open circles). maximum and zero molecular weight. This peak maximum equals the weight-average molecular weight which may be approximated by viscosityaverage molecular weight of the sample. Thus obtained differential curve by using the data of Table IIa is shown by open circles and the solid line in Figure 7. The distribution obtained by differentiating integral curve constructed from the same data by using Schulz-Dinglinger procedure is shown by chain line. Close agreement is observed between the two curves. Thus the usual Schulz-Dinglinger procedure to construct the molecular weight distribution of a polymer from fractionation data of the elution method or precipitation chromatography may successfully be applied to those of broad distribution such as prepared by free radical polymerization.

Acknowledgment. The present author wishes to thank Professor J. Isemura, Director of Protein Research Institute of Osaka University, for his permission to use ultracentrifuge, Drs. M. Matsumoto and Y. Ohyanagi of this laboratory and Professor H. Fujita of Osaka University for their interest and encouragement, and Mr. N. Sogabe for his help in fractionation.

\section{REFERENCES}

1. C. A. Baker and R. J. P. Williams, J. Chem. Soc., 2352 (1956).

2. N. S. Schneider, J. D. Loconti, and L. G. Holmes, J. Appl. Polym. Sci., 5, 354 (1961).

3. R. W. Hall, in "Techniques of Polymer Characterization" P. W. Allen., Ed., Butterworth, London, (1959), pp. 51-52.

4. J. E. Guillet, R. L. Combs, D. F. Slonaker, and H. W. Coover, Jr., J. Polym. Sci., 47, 307 (1960).

5. A. S. Keyon and I. O. Sayer, J. Polym. Sci., 43, 427 (1960).

6. M. J. R. Cantow, R. S. Porter, and J. F. Johnson, J. Appl. Polym. Sci., 8, 2963 (1964).

7. G. V. Sculz and A. Dinglinger, Z. Phys. Chem., B43, 47 (1936).

8. T. Homma, K. Kawahara, H. Fujita, and M. Ueda, Makromol. Chem., 67, 132 (1963).

9. M. Ueda, ibid., 90, 139 (1966).

10. The Committee on Molecular Weight and Molecular Weight Distribution of the Society of Polymer Science, Japan, (Chairman, Prof. A. Kotera), Reports on Prog. in Polymer Phys. in 
Japan, 9, 557 (1966).

11. J. L. Jungnickel and F. T. Weiss, J. Polym. Sci., 49, 437 (1961).

12. F. Wenger, Makromol. Chem., 37, 143 (1960).

13. K. Kawahara, ibid., 73, 1 (1964).

14. C. W. Pyun, and M. Fixman, J. Chem. Phys., 41, 937 (1964).

15. K. Kawahara, Kobunshi Kagaku (Chem. High Polymers), 18, 687 (1961).

16. J. Korai, R. Ullma, and F. R. Dirich, J. Phys. Chem., 62, 541 (1958).

17. T. Alfrey, A. Batovics, and H. Mark, J. Amer. Chem. Soc., 65, 2319 (1943).

18. see for example, F. Patat, E. Killmann and C. Schliebener, Fortschr. Hochpolym. -Forsch., 3, 332 (1964).

19. M. Ueda, unpublished results.

20. W. I. Patnode, US Patent 2306222 (1942).

21. H. W. McCormick, J. Polym. Sci., 36, 341 (1959).

22. G. C. Berry, ibid., Part B, 4, 161 (1966).

23. G. V. Schulz, Z. Physik. Chem., B46, 137 (1940); ibid., B47, 155 (1940).

24. M. L. Huggins, H. Okamato in "Polymer Fractionation," M. J. R. Cantow, Ed., Academic
Press, New York, N. Y., 1967, Chapter A.

25. M. Ueda, Kobunshi Kagaku (Chem. High Polymers), in press.

26. M. Matsumoto, and Y. Ohyanagi, ibid., 11, 7 (1954).

27. R. L. Scott, Ind. Eng. Chem., 45, 2532 (1953).

28. R. Koningsveld, Advan. Polym. Sci., 7, 1 (1970).

29. K. Kamide, K. Sugamiya, Abstracts, SPSJ 19th Symposium on Macromolecules, Kyoto, Octber 21, 1970.

30. P. J. Flory, "Principles of Polymer Chemistry," Cornell University Press, Ithaca, N. Y., 1953, p. 560 .

31. G. V. Schulz, Z. phys. Chem., B43, 25 (1939).

32. T. Fujimoto, N. Ozaki, and M. Nagasawa, $J$. Polym. Sci., Part A, 3, 2259 (1965).

33. M. Ueda, unpublished results.

34. A. Yamamoto, I. Noda, and M. Nagasawa, Polymer J., 1; 304 (1970).

35. S. R. Caplan, J. Polym. Sci., 35, 409 (1959).

36. W. R. Krigbaum and J. E. Kurz, ibid, 41, 275 (1959).

37. J. C. Moore, ibid., Part A, 2, 835 (1964). 